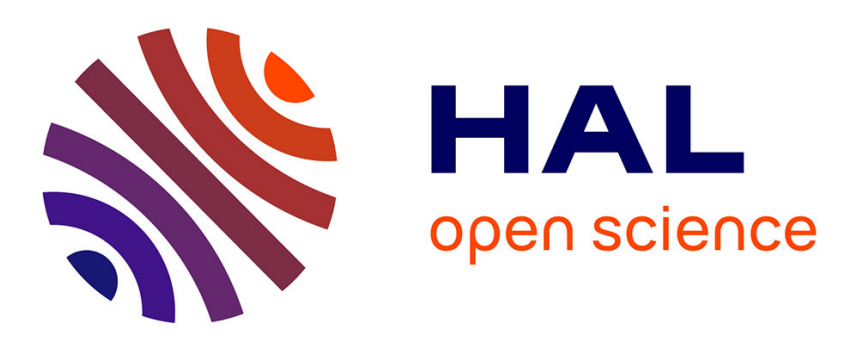

\title{
SNAREpin Assembly: Kinetic and Thermodynamic Approaches
}

Feng Li, Frédéric Pincet

\section{To cite this version:}

Feng Li, Frédéric Pincet. SNAREpin Assembly: Kinetic and Thermodynamic Approaches. Rutilio Fratti. SNAREs. Methods and Protocols, 1860, Springer, pp.71-93, 2019, Methods in Molecular Biology, 978-1-4939-8759-7. 10.1007/978-1-4939-8760-3_5. hal-01954666

\section{HAL Id: hal-01954666 https://hal.sorbonne-universite.fr/hal-01954666}

Submitted on 13 Dec 2018

HAL is a multi-disciplinary open access archive for the deposit and dissemination of scientific research documents, whether they are published or not. The documents may come from teaching and research institutions in France or abroad, or from public or private research centers.
L'archive ouverte pluridisciplinaire $\mathbf{H A L}$, est destinée au dépôt et à la diffusion de documents scientifiques de niveau recherche, publiés ou non, émanant des établissements d'enseignement et de recherche français ou étrangers, des laboratoires publics ou privés. 
SNAREpin Assembly: Kinetic and Thermodynamic Approaches

$$
\text { Feng } \mathrm{Li}^{1,{ }^{1,}} \text { and Frederic Pincet }{ }^{1,2, *}
$$

${ }^{1}$ Department of Cell Biology and Nanobiology Institute, School of Medicine, Yale University, 333 Cedar Street, New Haven, CT 06520, USA

${ }^{2}$ Laboratoire de Physique Statistique, Ecole Normale Supérieure, PSL Research University, Université Paris Diderot Sorbonne Paris Cité, Sorbonne Universités UPMC Univ Paris 06, CNRS, Paris, France.

*Correspondence to: Feng Li (feng.li@yale.edu) and Frederic Pincet (pincet@lps.ens.fr) 


\begin{abstract}
Proteins constantly interact and often form molecular complexes. The dynamics of most biological processes strongly rely on the kinetics \& thermodynamics of assembly and disassembly of these complexes. Consequently an accurate characterization of these kinetics and thermodynamics that underlie them provides key information to better understand these processes. Here, we present two efficient techniques to quantify the assembly and disassembly of protein complexes: Isothermal Titration Calorimetry and Fluorescence Anisotropy. As an example we focus on the formation of SNAREpins and also present how to prepare SNARE proteins to use in these experimental setups. We then show how to use these techniques to determine the critical factors that activate assembly kinetics.
\end{abstract}

Key words: Isothermal Titration Calorimetry, Fluorescence Polarization, Fluorescence Anisotropy, SNARE, VAMP2, Syntaxin, SNAP25, protein assembly, protein interaction 


\section{Introduction}

Living systems are very dynamic with constant assembly and disassembly of molecular complexes that either provide a signal or perform a specific task. For instance, soluble Nethylmaleimide-sensitive factor attachment protein receptors (SNAREs) form a family of proteins that provide the fuel for membrane fusion in most intra-cellular trafficking (1-4). SNAREs achieve the fusion of a vesicle with a flat membrane in order to deliver cargos outside of a compartment (organelle or cell). An archetypical example is the fusion of synaptic vesicle with the neuronal presynaptic plasma membrane to deliver neurotransmitters at the synapse during neurotransmission (5-8). Initially, one SNARE is anchored the synaptic vesicle membrane, the vSNARE VAMP2, and another one is anchored in the target plasma membrane, the t-SNARE which is a heterodimer composed of Syntaxin1A and SNAP25. When the two SNAREs contact, they zipper from their $\mathrm{N}$-terminal region to their $\mathrm{C}$-terminal region and form a four helix bundle coiled-coil, the SNAREpin $(9,10)$. Upon zippering of the SNAREpin the two membranes are force to come in very close contact and to eventually fuse (11-13).

Studies show that zippering between the $\mathrm{v}$ - and t-SNAREs is intrinsically step-like $(14,15)$. The SNAREs start their initial association at their N-termini and form an intermediate, partially zippered complex, waiting for the signal of action potential. Upon $\mathrm{Ca}^{2+}$ entry, the $\mathrm{C}$-terminal subdomains of the SNAREs further zipper up rapidly and provide energy to bring the two membranes even closer and trigger bilayer merging and content release in sub-millisecond timescale $(7,14)$. After the vesicles release their cargo, a new batch of vesicles docks to the presynaptic membrane and their v-SNAREs associate with t-SNAREs to be primed for the next round of fusion $(16,17)$. At fast synapses, the rate of arriving of new, ready-to-fuse vesicles is $\sim 100 \mathrm{~ms}$ (18-20). The assembly of the fusion-competent protein complex includes the N-terminal association of the $\mathrm{t}$ - and v-SNAREs, and thus this association must occur within such a timeframe $(5,15,21-24)$. Therefore, both $\mathrm{N}$ - and C-terminal assemblies of the SNAREs are required to sufficient fast to accommodate the kinetics of vesicle docking, priming and fusion.

Here, our goal is to demonstrate how to characterize the kinetics of assembly of SNAREpins by using two experimental setups, Isothermal Titration Calorimetry (ITC) and Fluorescence Anisotropy. We will work only with the cytosolic parts of the proteins which are involved in the zippering process that leads to the SNAREpin formation. First we will describe the various 
plasmids that we used and how to express and purify the corresponding proteins. Then, we will focus on the two techniques.

ITC is a technique that is designed to measure the thermodynamic parameters of a wide variety of molecular interactions(25). During an ITC measurement, the first reactant is stored in its sample cell, while the pipet holds the second reactant and injects a small volume of it into the sample cell each time in a titration manner. The heat that is either released or absorbed during the reaction of these two reactants in each injection is measured. ITC is mostly used to study the binding reactions of small molecules with biomacromolecules in solution, as well as interactions between soluble DNA, RNA and cytosolic proteins $(8,24)$. It may also be able to study transmembrane proteins that can be reconstituted on liposomes, nanodiscs, or bicelles through their transmembrane domains $(14,26)$. Here we will show the example of the titrations of various cytosolic VAMP2 domains into t-SNARE.

Fluorescence anisotropy is based on the fact that polarization anisotropy of fluorescence strongly depends on the weight and dimensions of the molecular complex or entity (e.g. liposome) that the dye is bound to $(27,28)$. A free dye will have limited fluorescence polarization anisotropy while a dye bound to a liposome or a large molecule will have much larger fluorescence anisotropy (29). Hence the principle of this technique is to mix two solutions, the first one contains the dye bound to a small molecule (here part of VAMP2) and the second one contains the much heavier tSNARE (possibly on liposome if it includes the transmembrane domain). As VAMP2 domains bind to t-SNARE, their fluorescence polarization anisotropy increases. It can be measured in realtime which directly shows the kinetics of assembly of the molecular complex. Fluorescence anisotropy has often been used to qualitatively compare the kinetics of reaction at different conditions (30). Here we present the theory and equations which are able to quantify the measurements and directly determine kinetic parameters such as the on-rate, $k_{o n}$ and the off-rate, $k_{o f f}$, and thermodynamic parameter such as affinity constant, $K_{D}(14,24)$.

\section{Materials}

\subsection{DNA Plasmids}

1. Cytosolic t-SNARE complex (CDT). Clone of the cytoplasmic domain of rat Syntaxin1A (residues 1-265) into a pET28a vector whose N-terminal 6xHis tag has been deleted to make the plasmid for soluble Syntaxin1A, as described before (11-13). 
Clone mouse SNAP25B (residues 1-206) into a pET15 vector which contains an Nterminal $6 \mathrm{xHis}$ tag to make the plasmid for SNAP25.

2. Cytosolic VAMP2 (CDV). Clone mouse VAMP2 into a pET SUMO vector containing $\mathrm{N}$-terminal $6 \mathrm{xHis}$ tag to produce the plasmid for CDV. The construct contains VAMP2 residues 1 to 94 , as previously described $(11,12,14)$. Use the T4 ligase to place the DNA insert into the vector through BamHI and NotI restriction sites.

3. Cytosolic VAMP2 (CDV-S28C). Point mutate the cytosolic VAMP2 plasmid above at position 28 to produce the plasmid for CDV-S28C. Use QuikChange Site-Directed Mutagenesis kit and follow the manufacturer's instruction to mutate Ser at position 28 to Cys, as described before $(11,12)$.

4. $N$-terminal subdomain of VAMP2 $(V n)$. Clone the N-terminus of mouse VAMP2 into a pCDFDuet-1 vector containing N-terminal GST-PreScission to produce the plasmids for the Vn. The Vn constructs are: Vn (VAMP2 residues 28 to 57), and Vn-S28C (VAMP2 residues 1 to 57 , with a single Cysteine mutation at position 28, as previously described $(14,24,31)$.

5. C-terminal subdomain of VAMP2 $(V c)$. Clone the C-terminus of mouse VAMP2 into a pET SUMO vector containing N-terminal 6xHis tag to produce the plasmid for Vc. The construct contains VAMP2 residues 58 to 94 with a single Cysteine residue in the end of the sequence, as previously described (14).

\subsection{Expression and Purification of Proteins}

\section{HEPES}

2. IPTG, Isopropyl $\beta$-D-thiogalactoside

\section{Ampicillin}

4. Kanamycin

5. Imidazole

6. Glycerol

7. DTT 
8. Pierce ${ }^{\mathrm{TM}}$ TCEP-HCl

9. n-Octyl- $\beta$-d-glucopyranoside (OG)

10. Bio-Beads SM-2 adsorbents

11. Pierce ${ }^{\mathrm{TM}}$ Glutathione Agaros

12. Ni-NTA agarose

13. PreScission Protease

14. SUMO protease

15. HiLoad Superdex 200 16/600 GL prepacked column

16. Superdex 75 10/300 GL prepacked column

17. Amicon ${ }^{\circledR}$ Ultra-4 Centrifugal Filter Units, $3 \mathrm{kDa}$ and $10 \mathrm{kDa}$ NMWL

18. Gas-tight glass syringe (Hamilton)

19. D-Tube ${ }^{\mathrm{TM}}$ Dialyzer Midi, 6-8 kDa MWCO

20. D-Tube ${ }^{\mathrm{TM}}$ Dialyzer Midi, MWCO 3.5 kDa

21. Lysis buffer for protein purification: $25 \mathrm{mM}$ HEPES at $\mathrm{pH} 7.4,400 \mathrm{mM} \mathrm{KCl}, 10 \%$ glycerol, $0.5 \mathrm{mM}$ TCEP, $0.5 \mathrm{mM}$ PMSF, and dissolved protease inhibitor cocktail. Prepare stock solution of PMSF by dissolving its power in $100 \%$ ethanol to a final concentration of $100 \mathrm{mM}$. Add $250 \mu \mathrm{L}$ PMSF stock solution into $50 \mathrm{~mL}$ lysis buffer while the buffer was under vigorous stirring using a magnetic bar. Then add one tablet of SIGMAFAS EDTA-free protease inhibitor cocktail into the lysis buffer. Keep the buffer under vigorous stirring in the cold room until the tablet was completely dissolved

22. Wash buffer for protein purification: $25 \mathrm{mM}$ HEPES at $\mathrm{pH} 7.4,400 \mathrm{mM} \mathrm{KCl}, 10 \%$ glycerol, and $0.5 \mathrm{mM}$ TCEP.

23. Cleavage buffer for protein purification: $25 \mathrm{mM}$ HEPES at $\mathrm{pH} 7.4,150 \mathrm{mM} \mathrm{KCl}, 10 \%$ glycerol, $0.5 \mathrm{mM}$ TCEP, and $30 \mathrm{mM}$ Imidazole.

24. PBS buffer: $137 \mathrm{mM} \mathrm{NaCl}, 3 \mathrm{mM} \mathrm{KCl}, 10 \mathrm{mM}$ sodium phosphate dibasic, $2 \mathrm{mM}$ potassium phosphate monobasic. 


\subsection{Isothermal Titration Calorimetry}

1. MicroCal iTC200 (Malvern)

2. MicroCal ThermoVac Sample Degassing Thermostat (Malvern)

3. Cleaning Device for iTC200 using ThermoVac, ITC200 (Malvern)

4. Plastic coated Filling Syringe for ITC200 (Malvern)

\subsection{Fluorescence Anisotropy assay}

1. PC1 Photon Counting Spectrofluorimeter (ISS)

2. Four-cell sample chamber with accurate temperature control (ISS)

3. Hellma Quartz Cuvettes (Hellma)

4. Longpass filter $625 \mathrm{~nm}$ (Edmund Optics)

5. Neutral density filters $625 \mathrm{~nm}$ (Edmund Optics)

6. Texas Red ${ }^{\mathrm{TM}} \mathrm{C} 2$ Maleimide

7. PD MidiTrap G-25 Sample Preparation Columns

\section{Methods}

\subsection{Protein expression and purification}

\subsubsection{Cytosolic t-SNARE complex (CDT).}

1. Transformation: Thaw $30 \mu \mathrm{L}$ BL21-Gold DE3 competent cells on ice. Add $5 \mu \mathrm{L}$ plasmid for soluble Syntaxin1A and $5 \mu \mathrm{L}$ SNAP25 plasmid into the cells, and incubate 45 minutes on ice. Heat shock the cells for 30 seconds in $42^{\circ} \mathrm{C}$ water bath. Add $150 \mu \mathrm{L}$ S.O.C. medium into the cells, place them in the $37^{\circ} \mathrm{C}$ incubator and shake at $0.4 \mathrm{~g}(220 \mathrm{rpm})$ for 1 hour. Spread the cells on a LB agar plate with $50 \mu \mathrm{g} / \mathrm{mL}$ Kanamycin and $100 \mu \mathrm{g} / \mathrm{mL}$ Ampicillin. Incubate the agar plate overnight at $37^{\circ} \mathrm{C}$.

2. Cell culture: use the tip of a $10 \mathrm{uL}$ pipette to contact a single colony on the agar plate and transfer the cells into $5 \mathrm{~mL}$ autoclaved LB medium with $50 \mu \mathrm{g} / \mathrm{mL}$ Kanamycin and $100 \mu \mathrm{g} / \mathrm{mL}$ Ampicillin. Incubate and shake the medium at $37^{\circ} \mathrm{C}$ for 4 hours. Transfer the medium into $250 \mathrm{~mL}$ autoclaved LB medium with $50 \mu \mathrm{g} / \mathrm{mL}$ Kanamycin and $100 \mu \mathrm{g} / \mathrm{mL}$ Ampicillin. Incubate and shake the medium at $37^{\circ} \mathrm{C}$ overnight. 
Prepare $4 \mathrm{~L}$ autoclaved LB medium with $50 \mu \mathrm{g} / \mathrm{mL}$ Kanamycin and $100 \mu \mathrm{g} / \mathrm{mL}$ Ampicillin in the next morning, $2 \mathrm{~L}$ in each flask. Transfer $100 \mathrm{~mL}$ of the overnight culture to each flask. Incubate and shake $(5 \mathrm{~g}, 220 \mathrm{rpm})$ the cultures at $37^{\circ} \mathrm{C}$ until $\mathrm{OD}$ reaches 0.6 to 0.8 . Add IPTG to the cultures, final concentration of IPTG is $0.5 \mathrm{mM}$. Add $2 \mathrm{~mL}$ Ampicillin at $100 \mathrm{mg} / \mathrm{mL}$ to each flask. Incubate and shake (5 g, $220 \mathrm{rpm}$ ) the cultures at $37^{\circ} \mathrm{C}$ for 3 to 4 hours. Harvest the cells by spinning down them at 6,060 g. Remove the supernatant and store the cell pellets in $-80^{\circ} \mathrm{C}$ freezer.

3. Lysis: Prepare and pre-chill $50 \mathrm{~mL}$ lysis buffer. Thaw and re-suspend the cell pellets in the pre-chilled lysis buffer. Pass the bacterial cells through a $191 / 2$ G needle. Pass the bacterial cells through the French press cell disruptor 4 times. Centrifuge the lysate at $186,000 \mathrm{~g}$ in a Ti45 rotor (Beckman) for 50 minutes at $4^{\circ} \mathrm{C}$. After ultracentrifugation, the supernatant of the samples is very clear. Transfer the supernatant from the centrifugal tube to a polypropylene centrifuge bottle with conical bottom.

4. Wash the Ni beads: take one $50 \mathrm{~mL}$ centrifugal tubs. Add $8 \mathrm{~mL}$ Ni NTA agarose suspension ( $4 \mathrm{~mL}$ pure Ni beads). Then add $\sim 30 \mathrm{~mL}$ wash buffer into the tube, mixing by inverting and vortex. Centrifuge in the cold room at $500 \mathrm{~g}$ for 4 minutes. Then remove supernatant by pipet. Add $25 \mathrm{~mL}$ wash buffer to the tube again, mix by inverting and vortex, centrifuge in the cold room at $500 \mathrm{~g}$ for 4 minutes. Remove supernatant by pipet. Repeat for two more times. Remove supernatant by pipet, and then add $\sim 4 \mathrm{~mL}$ buffer and mix. Finally the slurry with 1:1 bead/buffer ratio.

5. Binding to affinity beads: Add $6 \mathrm{~mL}$ pre-washed Ni-NTA slurry into the supernatant. Put the conical bottle on the spin wheel in the cold room and spin overnight. Rinse and pre-hill a BioRad Econo-Pac ${ }^{\circledR}$ chromatography column in the cold room. Transfer the supernatant and Ni-NTA beads into the column. Discard the flowthrough, and collect the Ni-NTA beads in the column. Rinse the beads with $20 \mathrm{~mL}$ wash buffer supplemented with $50 \mathrm{mM}$ Imidazole five times. Rinse the beads one more time with $20 \mathrm{~mL}$ cleavage buffer. Keep about $4 \mathrm{~mL}$ buffer above the beads.

6. Protease cleavage and elution: add 300 units of Thrombin into the Ni-NTA slurry. Seal both ends of the column and put the column on the spin wheel in the cold room and spin at the lowest speed overnight. Add $300 \mu \mathrm{L}$ pre-washed Benzamidine Sepharose 4 Fast Flow (high sub) slurry into the column and spin for 3 hours. Elute 
the protein solution from the column using gravity. Further purify the protein by size exclusion chromatography on a HiLoad Superdex 200 column using the ÄKTA ${ }^{\text {TM }}$ chromatography system.

7. Characterization: test the purity of the protein using SDS-PAGE analysis. Measure the concentration of the protein by Bradford protein assay with BSA as the standard.

3.1.2 Cytosolic VAMP2 (CDV), Cytosolic VAMP2 (CDV-S28C), and C-terminal subdomain of VAMP2 (Vc).

Expression and purification of these proteins were similar to the protocol above with small differences. Use SUMO protease instead of Thrombin to cleave the N-terminal 6xHis-SUMO tag, similar to that described before $(8,11,12,14,24)$. Add $100 \mu \mathrm{L} 3$ $\mathrm{mg} / \mathrm{mL}$ SUMO protease into the Ni-NTA slurry. Seal both ends of the column and put the column on the spin wheel in the cold room and spin at the lowest speed overnight. Add $300 \mu \mathrm{L}$ extra Ni-NTA slurry into the column and spin for 3 hours to remove the SUMO protease. Elute the protein solution from the column using gravity. Further purify the protein by size exclusion chromatography on a HiLoad Superdex 75 column using the ÄKTA ${ }^{\mathrm{TM}}$ chromatography system.

\subsubsection{N-terminal subdomain of VAMP2 (Vn and Vn-S28C).}

Expression and purification of these proteins were similar to the protocol above with small differences. Use PreScission protease instead of Thrombin to cleave the Nterminal GST tag, similar to that described before $(12,14,24,31)$. Add $200 \mu \mathrm{L} 1.5$ $\mathrm{mg} / \mathrm{mL}$ PreScission protease into the Ni-NTA slurry. Seal both ends of the column and put the column on the spin wheel in the cold room and spin at the lowest speed overnight. Add $300 \mu \mathrm{L}$ extra Ni-NTA slurry into the column and spin for 3 hours to remove the PreScission protease. Elute the protein solution from the column using gravity. Further purify the protein by size exclusion chromatography on a HiLoad Superdex 75 column using the ÄKTA ${ }^{\mathrm{TM}}$ chromatography system.

\subsection{Isothermal Titration Calorimetry (ITC) measurements}

1. Before ITC experiments, purify the t-SNARE, Vn, Vc, and CDV by gel filtration using a regular or HiLoad Superdex 75 or 200 column (GE Healthcare Life Sciences) and PBS with $0.25 \mathrm{mM}$ TCEP as the running buffer, respectively. Pool peak fractions, 
and then concentrate them using Amicon ${ }^{\circledR}$ ultra-4 centrifugal filter units. Concentrate the t-SNARE to around $50 \mu \mathrm{M}$, while concentrating Vn, Vc, and CDV to around 600 $\mu \mathrm{M}$.

2. Transfer the t-SNARE and Vn, Vc, and CDV into four D-Tube ${ }^{\mathrm{TM}}$ midi dialyzer tubes, respectively, and then dialyze them in the same flask against 4 liters of PBS buffer with $0.25 \mathrm{mM}$ TCEP for 4 hours at $4^{\circ} \mathrm{C}$ and then dialyzed against another 4 liters of fresh PBS buffer with $0.25 \mathrm{mM}$ TCEP overnight at $4^{\circ} \mathrm{C}$ (see Note 1).

3. Determine the concentrations of dialyzed proteins by using the Thermo Scientific Pierce Bicinchoninic Acid (BCA) protein assay kit with BSA as the standard (see Note 2).

4. Use the accessory station and pre-installed program to clean the syringe on the titration pipet. Select "clean the syringe long" to do the cleaning twice. Clean the reference cell with water using the plastic-coated syringe five times. Fill the reference cell with MilliQ water. Use the ThermoVac to clean sample cell with $100 \mathrm{~mL}$ MilliQ water. The sample cell and pipet need to be cleaned after each use (see Note 3).

5. Use the plastic-coated syringe to transfer about $200 \mu \mathrm{L}$ MilliQ water to the sample cell. Aspirate about $60 \mu \mathrm{L}$ MilliQ water from the eppendorf to the syringe on the pipet. Purge 3 times to remove air bubbles. Perform a water-water titration to check the noise and stability of baseline (Figure 2).

6. Pre-estimate $\Delta H$ and affinity constant $K_{D}$ of the reaction to be study with the ITC. Use the pre-install simulation software to estimate the concentrations of reactant in the sample cell and the titrant in the titration pipet. Dilute the protein proteins to the corresponding concentrations with the exactly same buffer used in the dialysis.

7. Perform ITC experiments with a Microcal ITC 200 instrument similarly to that was described before $(8,14,24,32)$. Typically, load about $200 \mu \mathrm{L}$ of t-SNARE solution into the sample cell, and load about $60 \mu \mathrm{L}$ of $\mathrm{Vn}$ (or Vc or CDV) solution into the titration pipet (see Note 4).

8. Set the reference power at 5. Set number of injections as 20 and $2 \mu \mathrm{L}$ per injection. Set the interval between injections as 5 minutes. Start titration.

9. Measure the power as function of time during the titration. This power-time curve records both the thermal baseline and the heat released or absorbed by the reaction in 
each injection. Use the pre-installed analysis software to subtract the baseline from the record thermograph and determine the power generated from each injection. The large power spikes represent the signal of binding reaction (Figure 3A and 3B upper panel). If the power spikes have similar amplitude as in water-water titration (Figure 3C), it usually indicates that no binding signal was detected (see Note 5).

10. Use Microcal Origin ITC200 software package to analyze the titration calorimetric data and obtain the thermodynamic parameters of the reaction, i.e., the stoichiometric number $(N)$, the molar binding enthalpy $(\Delta H)$, and the association constant $\left(K_{a}\right)$. Integrate the heat change from each injection, and then normalize it by the moles of protein in the injection. Assume a simple one site chemical reaction and use a nonlinear least squares fit to obtain the reaction equilibrium constant $\left(K_{a}\right)$ and the binding enthalpy $(\Delta H)$. Use the following thermodynamic equations to calculate the equilibrium dissociation constant $\left(K_{D}\right)$, the binding free energy $(\Delta G)$, and the binding entropy $(\Delta S)$ (see Note 6):

$$
K_{D}=1 / K_{a} \quad \text { and } \quad \Delta G=\Delta H-T \Delta S=-R T \ln \left(K_{a}\right)
$$

11. To investigate the molecular basis of the kinetically unfavorable $\mathrm{N}$-terminal assembly of the SNAREs, we pre-incubate t-SNARE with Vc peptide (molar ratio t-SNARE:Vc $\sim 1: 8)$. Vc pre-binds the t-SNARE and forms a partial complex at their C-termini, leaving the N-terminus of the t-SNARE available for Vn assembly. After the prebinding reaches thermodynamic equilibrium, load this t-SNARE/Vc mixture into the ITC cell and titrate it with Vn peptide (Figure 3D). Large heat spikes due to Vn assembling with the t-SNARE are measured during the initial injections. The thermodynamic parameters for this binding reaction are $\Delta H=-20.2 \mathrm{kcal}^{\circ} \mathrm{mol}^{-1}$ and $\mathrm{KD}_{D}$ $=7 \pm 1 n M$. Therefore, the pre-binding of $\mathrm{Vc}$ to the t-SNARE accelerates the $\mathrm{N}$ terminal assembly of SNAREs.

12. To test whether this pre-binding induced activation is limited to Vn peptide, we use the cytosolic domain of the v-SNARE (CDV, VAMP2 residue 1 to 94) to perform the experiments. In ITC measurement, we pre-incubate the t-SNARE with Vc peptide, and then titrate CDV into this mixture (Figure 3E). The thermodynamic parameters of this reaction are $K_{D}=15 \pm 2 \mathrm{nM}$ and $\Delta H=-20.2 \mathrm{kcal} \cdot \mathrm{mol}^{-1}$ (see Note 7). 


\subsection{Fluorescence Anisotropy assays}

1. Label CDV-S28C, Vn-S28C, and Vc-Cys mutants with Texas Red ${ }^{\circledR}$ C2 Maleimide (Life Technologies), similarly to previous report $(14,24)$. First reduce the proteins by incubating with $4 \mathrm{mM}$ TCEP for 30 minutes at $4^{\circ} \mathrm{C}$ with gentle rotation, and centrifuge them at $18,000 \mathrm{~g}$ for 20 minutes at $4^{\circ} \mathrm{C}$ to remove any precipitation. Add fluorescence dye into the protein solution at dye:protein $=3: 1$ molar ratio and incubate the mixture for 1 to 2 hours at room temperature with gentle rotation. Remove unreacted dye by passing through the PD MidiTrap G-25 column (GE Healthcare) three times.

2. Use the PC1 photon counting spectrofluorimeter (ISS) to measure fluorescence anisotropy. Use T-format polarization with a $625 \mathrm{~nm}$ long-path filter on the leftemission channel and a monochromator on the right-emission-channel. Control the temperature of sample chamber with $\pm 0.1^{\circ} \mathrm{C}$ accuracy. For Texas-Red labeled protein, the excitation wavelength was $580 \mathrm{~nm}$ and the emission wavelength at the rightemission-channel was $612 \mathrm{~nm}$. Use quartz cuvette (Hellma) for all experiments.

3. For kinetic studies, introduce the Texas-Red labeled cytosolic CDV-S28C, Vn-S28C, or Vc-Cys solution to a quartz cuvette (Hellma) with continuous and rapid magnetic stirring. Record anisotropy as a function of time. Inject cytosolic t-SNARE at various concentrations into the cuvette and mix them rapidly. Plot the data as anisotropy versus time, and set the beginning of mixing as time zero (Figure 4A, 4B and 4C).

4. To obtain the kinetics and thermodynamics parameters, we consider the following binding reaction:

$$
V+T \leftrightharpoons V T
$$

The kinetics equation is

$$
\frac{d[V T]}{d t}=k_{o n}[V][T]-k_{o f f}[V T]
$$

where $k_{o n}$ is the on-rate and $k_{\text {off }}$ is the off-rate, $[V],[T]$ and $[V T]$ are the concentration of VAMP2 peptide, t-SNARE and SNARE complex at time $t$, respectively.

Let $V_{0}$ and $T_{0}$ be the initial concentration (or total concentration) of VAMP2 peptide and t-SNARE, respectively, then

$$
V_{0}=[V]+[V T] \quad \text { and } \quad T_{0}=[T]+[V T]
$$


The measured anisotropy $A$ at time $t$ is an average of anisotropy of the fluorophores associated with VAMP2 peptide and the fluorophores associated with the SNARE complex. Let $A_{V}$ be the anisotropy of VAMP2 peptide (all of the fluorophores are associated with VAMP2), and $A_{V T}$ be the anisotropy of complex (all of the fluorophores are associated with the complex), then

$$
A=\frac{[V]}{[V]+[V T]} A_{V}+\frac{[V T]}{[V]+[V T]} A_{V T}=\left(1-\frac{[V T]}{V_{0}}\right) A_{V+}+\frac{[V T]}{V_{0}} A_{V T}
$$

thus,

$$
\frac{[V T]}{V_{0}}=\frac{A-A_{V}}{A_{V T}-A_{V}}
$$

Equation 1 can be written as,

$$
\frac{d[V T]}{d t}=k_{o n}\left(V_{0}-[V T]\right)\left(T_{0}-[V T]\right)-k_{o f f}[V T]
$$

At the initial stage of the binding reaction, $[V T]$ is close to zero. Hence, equation 3 can be simplified as

$$
\frac{d[V T]}{d t}=k_{o n} V_{0} T_{0}
$$

Combine equations 2 and 4, we obtain

$$
\frac{d A / d t}{\left(A_{V T}-A_{V}\right)}=k_{o n} T_{0}
$$

To obtain $k_{o n}$, we perform a series of reactions that labeled VAMP2 peptide binds to tSNARE at various initial concentrations, $T_{0}$, and monitored the variation of $A$ with $t$. For each $T_{0}$, we obtain the initial rate $d A / d t$ from the $A$ versus $t$ curve, then plot $(d A / d t) /\left(A V T-A_{V}\right)$ versus $T_{0}$, which is linear, and the slope gives $k_{o n}$ (Figure 4D). Here we observe that the striking difference is that Vn assembled with t-SNARE indeed much slower than Vc and CDV. The on-rates for Vc and CDV assembly are similar, 6,000 to $7,000 \mathrm{M}^{-1} \mathrm{~S}^{-1}$; whereas $\mathrm{Vn}$ assembles with t-SNARE at an on-rate of only about $500 M^{-1} S^{-1}$.

5. To obtain the affinity constants, we consider the phase where the reaction reaches equilibrium and the measured anisotropy reached plateau. Let $K_{D}$ be the affinity constant, $A_{p}$ be the measured anisotropy at equilibrium, and $[V]_{p},[T]_{p}$, and $[V T]_{p}$ be 
the concentration of VAMP2 peptide, t-SNARE and SNARE complex at equilibrium, respectively, then:

$$
\begin{aligned}
K_{D} & =\frac{[V]_{p}[T]_{p}}{[V T]_{p}}=\frac{\left(V_{0}-[V T]_{p}\right)\left(T_{0}-[V T]_{p}\right)}{[V T]_{p}} \\
A_{p} & =\left(1-\frac{[V T]_{p}}{V_{0}}\right) A_{V}+\frac{[V T]_{p}}{V_{0}} A_{V T}
\end{aligned}
$$

Solving equation 6 for $[V T]_{p}$, and then entering into equation 7 , we have

$$
A_{p}=A_{V}+\left(A_{V T}-A_{V}\right)\left\{\frac{\left(K_{D}+V_{0}+T_{0}\right)-\sqrt{\left(K_{D}+V_{0}+T_{0}\right)^{2}-4 V_{0} T_{0}}}{2 V_{0}}\right\}
$$

By changing the initial concentration of t-SNARE, $T_{0}$, while keeping $V_{0}$ constant, one can obtain a curve of $A_{p}$ as a function of $T_{0} . K_{D}$ is obtained using equation 8 , and applying a non-linear regression fit to the $A_{p}$ versus $T_{0}$ curve (33). The off-rate, $k_{o f f}$, can also be obtained by using $K_{D}=k_{\text {off }} / k_{\text {onn }}$. In Figure 4E, we are able to evaluate the affinity constants of $\mathrm{Vn}, \mathrm{Vc}$ and $\mathrm{CDV}$ binding to t-SNARE. These results show that $\mathrm{Vn}, \mathrm{Vc}$, and CDV are all able to bind t-SNARE with similar $K_{D}: 85 n M$ for $\mathrm{Vn}, 170$ $n M$ for $\mathrm{Vc}$, and $110 n \boldsymbol{M}$ for $\mathrm{CDV}$.

6. Fluorescence anisotropy is able to study how the kinetics of SNARE assembly is affected by pre-structuring of the t-SNARE. Incubate the t-SNARE and Vc at molar ratio t-SNARE: $\mathrm{Vc} \sim 1: 8$ for 60 minutes. Monitor the anisotropy of labeled $\mathrm{Vn}$ as it binds to t-SNARE that has been prebound with Vc (Figure 5A). Determine the on-rate of the assembly and obtain the affinity constant $K_{D}$ (Figure 5C). Similarly, we also determine the on-rate of labeled CDV assembling with the t-SNARE pre-bound with Vc using fluorescence anisotropy (see Note 8).

7. We are able to determine the energy barriers during $\mathrm{N}$ - and C-terminal assemblies of the SNAREs. Perform fluorescence anisotropy measurements to monitor labeled Vn peptide assembling with t-SNARE or t-SNARE preincubated with $\mathrm{Vc}$, at different temperatures $T$. Obtained the on-rate $k_{o n}$, affinity constant $K_{D}$, and the off-rate $k_{o f f}$ (through $K_{D}=k_{o n} / k_{o f f}$,) at each temperature. Use the classical Arrhenius' equation, $k=A e^{-E_{a} /\left(k_{B} T\right)}$, where $A$ is the pre-exponential factor, $k_{B}$ is the Boltzmann constant, to plot $k_{o n}$ as a function of $1 / T$ to obtain the activation barrier of the assembly reaction, $E_{a, \text { on }}$ (Figure 6A, see Note 9). 
8. Similarly, Plot $k_{\text {off }}$ as a function of $1 / T$ to obtain the activation barrier of the disassociation, $E_{a \text {, off }}$ (Figure 6B, see Note 10).

9. Determine the energy barrier of the C-terminal assembly of labeled Vc binding to tSNARE using the same approach. In addition, preincubate t-SNARE with a Vn peptide, and measured the anisotropy of labeled Vc during it assembling with the prebound t-SNARE, and obtained the energy barrier (Figure 6C and 6D, see Note 11). With the energy barriers of both $\mathrm{N}$ - and C-terminal assembly of the SNAREs, we are able to construct an energy landscape to account for the $\mathrm{N}$ - to $\mathrm{C}$ - directional zippering (Figure 7, see Note 12).

\section{Notes}

1. It is critical to make certain that the samples used in titration contain identical buffer. The purpose for buffer match is to ensure that the only difference between these samples is the presence of different proteins. If the samples have buffer mismatch, the extra heat corresponding to the dilution of unmatched components will be integrated into the heat associated with protein-protein interaction. Sometimes the dilution heat can be large and it can introduce large error to quantification of the protein-protein interaction. Extensive dialysis is required to avoid buffer mismatch.

2. It is essential to have an accurate measurement of the concentrations of the proteins used in the ITC. Errors in protein concentrations will impair the reliability of the thermodynamic parameters that are determined from ITC, or sometimes it may even give misleading conclusion. There are several methods to measure protein concentration. A simple and direct assay method is to measure its UV absorbance at $280 \mathrm{~nm}$. Amino acids such as phenylalanine, tryptophan, and tyrosine strongly absorb UV light. Hence protein containing one or more of these aromatic side chains can be accurately measured using this method. There are several reagent-based colorimetric assays. The bicinchoninic acid assay (BCA) uses bicinchoninic acid to chelate $\mathrm{Cu}^{+}$ which has been reduced by the peptide bonds in the protein, producing a complex that strongly absorbs light at $562 \mathrm{~nm}(34,35)$. The Bradford assay uses Coomassie dye to bind the protein. This binding stabilizes the blue form of the Coomassie dye and induces a brown-to-blue color shift, which can be quantified by measuring absorption at $595 \mathrm{~nm}$ (36). No single method can be considered to be the ideal for determining 
protein concentration. Proper method needs to be selected depending on the protein sample. Usually a standard calibration curve using BSA at various concentrations is needed for each protein assay.

3. It is critical to make sure the ITC is properly and thoroughly cleaned. Usually it is sufficient to clean the syringe on the pipet with ITC's accessory station and preinstalled program. However, sometimes the accessory station and pre-install program cannot clean the sample cell good enough, especially when protein precipitates in the sample cell. We usually connect the sample cell to a vacuum aspirator collection bottle, and then use the ThermoVac to aspirate liquid from water source to the sample cell, then to the collection bottle. We use 100 to $200 \mathrm{~mL}$ to clean the sample cell. When sample cell is severely contaminated, it is necessary to incubate the sample cell with Contrad-70 at elevated temperature for 45 minutes before water flush.

4. If air bubble exists in either the sample cell or in the titration pipet, sharp spikes will appear randomly on the titration profile. Air bubbles are likely to form on the wall of the sample cell when the protein solution is not properly prepared or loaded into the cell. Degassing the sample help reducing air bubbles. Another useful method is to equilibrate the temperature of the sample to the same temperature of the cell before loading them into the instrument.

5. We separately titrate the C-terminal subdomain of v-SNARE (Vc peptide, VAMP2 residues 58 to 94) and the entire cytosolic domain (CDV, VAMP2 residues 1 to 94) into t-SNARE. The titration with Vc generates a large heat signal (Figure 3A), and the reaction between CDV and t-SNARE generates even large heat (Figure 3B). Surprisingly, when $\mathrm{Vn}$ is titrated into t-SNARE solution, the power spikes have similar amplitude as in water-water titration, suggesting that no binding signal has been detected (Figure 3C). This indicates that Vn does not bind to the t-SNARE during the time interval of titration.

6. Using a nonlinear least squares fit assuming a simple one site chemical reaction, we have obtained the thermodynamic parameters for the reactions in Figure 3. For Vc binding to the t-SNARE, the affinity constant, $K_{D}$, was about $440 \mathrm{nM}$ and $\Delta H$ of about $-19.1 \mathrm{kal}^{\mathrm{mol}} \mathrm{mo}^{-1}$ (Figure 3A). The reaction between CDV and t-SNARE gave $\Delta H$ about $-40.1 \mathrm{kcal} \cdot \mathrm{mol}^{-1}$ and $\mathrm{K}_{D}$ about $130 \mathrm{nM}$ (Figure 3B). 
7. It was noticeable that $\Delta H$ decreases from $-40.1 \mathrm{kcal} \cdot \mathrm{mol}^{-1}$ to $-20.2 \mathrm{kcal} \cdot \mathrm{mol}^{-1}$ upon prebinding of Vc (Table 1). This reduction was expected. In the absence of Vc prebinding, CDV assembled with the t-SNARE on both $\mathrm{N}$ - and C-termini; whereas in the presence of Vc pre-binding, assembly was initiated on the N-terminal subdomains of CDV and the t-SNARE, followed by displacement Vc and further assembly of their C-terminal subdomains. Consequently, $\Delta H$ corresponding to $\mathrm{C}$-terminal assembly canceled out. These ITC results in Figure 3 and Table 1 show that: (i) the N-terminal assembly is enthalpy driven and thermodynamically favorable, but kinetically unfavorable; (ii) pre-binding Vc to the t-SNARE accelerates the kinetics, but also improves the affinity of $\mathrm{N}$-terminal assembly.

8. As shown in Figure 4, the kinetics of Vn binding to t-SNARE is slow. Here when t-SNARE is prebound with $\mathrm{Vc}$, we find that the on-rate is increased by $\sim 1,000$ fold, to $4.9 * 10^{5} M^{-1} S^{-1}$ (Figure 5C). Hence the previous kinetically unfavorable N-terminal assembly became highly favorable when t-SNARE is prebound with Vc. We obtain the affinity constant from anisotropy measurements, $K_{D} \sim 5.6 n M$ (Figure 5D). Thus the binding affinity of N-terminal assembly is improved from $85 \mathrm{nM}$ to $5.6 \mathrm{nM}$ upon Vc addition. We have also determined the kinetics of labeled CDV assembling with the t-SNARE pre-bound with Vc using fluorescence anisotropy (Figure 5B). As the assembly is initialized on the N-termini of CDV and the t-SNARE, and progresses to their $\mathrm{C}$-termini to displace $\mathrm{Vc}$, we are able to directly measure the rate of $\mathrm{N}$-terminal assembly and obtain $k_{o n}=(5.1 \pm 1.0) * 10^{5} M^{-1} S^{-1}$ (Figure 5C), similar to previous report.(15) This rate is similar to the on-rate of Vn peptide binding with partially pre-assembled SNARE complex, suggesting that acceleration of $\mathrm{N}$-terminal assembly through pre-bound t-SNARE is a general feature. The affinity constant obtained from anisotropy measurements is about $K_{D}=1.2 \pm 0.3 n M$ (Figure 5D). Therefore, the pre-binding of Vc to the t-SNARE facilitates CDV assembly both kinetically and thermodynamically. The similarities in the kinetic and thermodynamic parameters between $\mathrm{Vn}$ and CDV assembling with t-SNARE suggest that, in both cases, activation originates from the same underlying molecular mechanism of pre-structuring of the t-SNARE. 
9. Synaptic vesicle fusion is driven by the C-terminal assembly of the SNAREs, while vesicle docking and priming result from their $\mathrm{N}$-terminal association. Thus determining energy barriers during $\mathrm{N}$ - and $\mathrm{C}$-terminal assembly is critical for understanding the kinetics of vesicle priming and fusion. We plot $k_{o n}$ as a function of $1 / T$ to obtain the activation barrier of the $\mathrm{N}$-terminal assembly reaction, $E_{a}$, on, both in the absence and presence of $\mathrm{Vc}$ prebinding (Figure 6A). The striking finding here is that the activation barrier of the N-terminal assembly is extremely high, $E_{a \text {, on }} \sim 34$ $k_{B} T$. This is the energy cost of prestructuring t-SNARE by opening the N-terminal 3helix bundle of t-SNARE to form a 4-helix bundle with the N-terminal subdomain of V-SNARE. This high energy barrier is the reason why the N-terminal assembly is intrinsically slow and needs to be accelerated. In contrast, when t-SNARE is prebound with $\mathrm{Vc}$, the energy barrier is substantially reduced to $E_{a, \text { on }} \sim 8.6 k_{B} T$. As shown above, this large energy reduction is because such prebinding already opens the $\mathrm{N}$ terminal 3-helix bundle into a 4-helix-bundle-like configuration, and this explains why $\mathrm{Vc}$ accelerates $\mathrm{N}$-terminal assembly.

10. As expected, the energy difference between the two states (Vn assembled state and

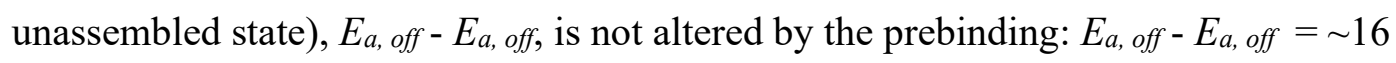
$k_{B} T$ both in the presence or absence of the prebinding. Hence prebinding of Vc only changes the activation energy.

11. We determine the energy barrier of the $\mathrm{C}$-terminal assembly by monitoring the anisotropy of labeled $\mathrm{Vc}$ binding to the t-SNARE. We obtain $E_{a, \text { on }} \sim 13 k_{B} T$ for the Cterminal association. We also preincubate t-SNARE with a Vn peptide, and measure the anisotropy of labeled Vc during its assembly with the prebound t-SNARE, and obtain $E_{a, \text { on }} \sim 4.7 k_{B} T$ (Figure 6C and 6D). Similarly, the energy barrier of C-terminal assembly was also largely decreased when t-SNARE is prebound. Prebinding Vn only changes the activation energies, but not the energy difference, which is about $18 k_{B} T$, between the Vc unbound and Vc bound states.

12. We construct an energy landscape to account for the $\mathrm{N}$ - to $\mathrm{C}$ - directional zippering using the activation barriers of both $\mathrm{N}$ - and $\mathrm{C}$-terminal assemblies (Figure 7). If $\mathrm{t}$ SNARE is not activated for the N-terminal assembly, the energy landscape of the zippering pathway is described by the blue line in Figure 7. The energy barrier is high, 
$34 k_{B} T$. When t-SNARE is activated upon prebinding with the C-terminal peptide of an R-SNARE, the energy landscape can be depicted by the black solid line in Figure

7. Here the barrier for $\mathrm{N}$-terminal association is largely reduced to $8.6 k_{B} T$, so that fast vesicle docking/priming becomes possible. Due to topological constraints, C-terminal zippering occurs after $\mathrm{N}$-terminal zippering, and hence the assembly substrate is the half-zippered SNARE intermediate. Therefore, the energy barrier of C-terminal assembly is reduced to $4.7 k_{B} T$ which allows rapid zippering to satisfy sub-millisecond fast fusion. There is an energy minimum between the two kinetic barriers, indicating the existence of the half-zippered SNARE intermediate (zippered on the N-termini but not on the C-termini).

\section{References}

1. Sollner, T., M. K. Bennett, S. W. Whiteheart, R. H. Scheller, and J. E. Rothman. 1993. A protein assemblydisassembly pathway in vitro that may correspond to sequential steps of synaptic vesicle docking, activation, and fusion. Cell 75:409-418.

2. Sollner, T., S. W. Whiteheart, M. Brunner, H. Erdjument-Bromage, S. Geromanos, P. Tempst, and J. E. Rothman. 1993. SNAP receptors implicated in vesicle targeting and fusion. Nature 362:318-324.

3. Rizo, J., and C. Rosenmund. 2008. Synaptic vesicle fusion. Nature structural \& molecular biology 15:665674.

4. Sudhof, T. C., and J. E. Rothman. 2009. Membrane fusion: grappling with SNARE and SM proteins. Science 323:474-477.

5. Jahn, R., and D. Fasshauer. 2012. Molecular machines governing exocytosis of synaptic vesicles. Nature 490:201-207.

6. Sudhof, T. C. 2012. The presynaptic active zone. Neuron 75:11-25.

7. Sørensen, J. B. 2009. Conflicting views on the membrane fusion machinery and the fusion pore. Annual review of cell and developmental biology 25:513-537.

8. Cho, R. W., D. Kummel, F. Li, S. W. Baguley, J. Coleman, J. E. Rothman, and J. T. Littleton. 2014. Genetic Analysis of the Complexin Trans-clamping Model for Cross-linking SNARE Complexes In Vivo. Proceedings of the National Academy of Sciences 111:10317-10322.

9. Sutton, R. B., D. Fasshauer, R. Jahn, and A. T. Brunger. 1998. Crystal structure of a SNARE complex involved in synaptic exocytosis at 2.4 angstrom resolution. Nature 395:347-353.

10. Fasshauer, D., R. B. Sutton, A. T. Brunger, and R. Jahn. 1998. Conserved structural features of the synaptic fusion complex: SNARE proteins reclassified as Q- and R-SNAREs. Proceedings of the National Academy of Sciences of the United States of America 95:15781-15786.

11. Li, F., F. Pincet, E. Perez, W. S. Eng, T. J. Melia, J. E. Rothman, and D. Tareste. 2007. Energetics and dynamics of SNAREpin folding across lipid bilayers. Nature structural \& molecular biology 14:890-896.

12. Li, F., F. Pincet, E. Perez, C. G. Giraudo, D. Tareste, and J. E. Rothman. 2011. Complexin activates and clamps SNAREpins by a common mechanism involving an intermediate energetic state. Nature structural \& molecular biology 18:941-946.

13. Wang, Y. J., F. Li, N. Rodriguez, X. Lafosse, C. Gourier, E. Perez, and F. Pincet. 2016. Snapshot of sequential SNARE assembling states between membranes shows that N-terminal transient assembly initializes fusion. Proc Natl Acad Sci USA doi: 10.1073/pnas. 1518935113:in press.

14. Li, F., D. Kummel, J. Coleman, K. M. Reinisch, J. E. Rothman, and F. Pincet. 2014. A Half-Zippered SNARE Complex Represents a Functional Intermediate in Membrane Fusion. Journal of the American Chemical Society 136:3456-3464. 
15. Walter, A. M., K. Wiederhold, D. Bruns, D. Fasshauer, and J. B. Sorensen. 2010. Synaptobrevin Nterminally bound to syntaxin-SNAP-25 defines the primed vesicle state in regulated exocytosis. The Journal of cell biology 188:401-413.

16. Sudhof, T. C. 1995. The synaptic vesicle cycle: a cascade of protein-protein interactions. Nature 375:645653.

17. Pyle, J. L., E. T. Kavalali, E. S. Piedras-Renteria, and R. W. Tsien. 2000. Rapid reuse of readily releasable pool vesicles at hippocampal synapses. Neuron 28:221-231.

18. Dittman, J. S., and W. G. Regehr. 1998. Calcium dependence and recovery kinetics of presynaptic depression at the climbing fiber to Purkinje cell synapse. The Journal of neuroscience : the official journal of the Society for Neuroscience 18:6147-6162.

19. Crowley, J. J., A. G. Carter, and W. G. Regehr. 2007. Fast vesicle replenishment and rapid recovery from desensitization at a single synaptic release site. The Journal of neuroscience : the official journal of the Society for Neuroscience 27:5448-5460.

20. Moser, T., and D. Beutner. 2000. Kinetics of exocytosis and endocytosis at the cochlear inner hair cell afferent synapse of the mouse. Proceedings of the National Academy of Sciences of the United States of America 97:883-888.

21. Hua, S. Y., and M. P. Charlton. 1999. Activity-dependent changes in partial VAMP complexes during neurotransmitter release. Nature neuroscience 2:1078-1083.

22. Xu, T., B. Rammner, M. Margittai, A. R. Artalejo, E. Neher, and R. Jahn. 1999. Inhibition of SNARE complex assembly differentially affects kinetic components of exocytosis. Cell 99:713-722.

23. Sorensen, J. B., K. Wiederhold, E. M. Muller, I. Milosevic, G. Nagy, B. L. de Groot, H. Grubmuller, and D. Fasshauer. 2006. Sequential N- to C-terminal SNARE complex assembly drives priming and fusion of secretory vesicles. The EMBO journal 25:955-966.

24. Li, F., N. Tiwari, J. E. Rothman, and F. Pincet. 2016. Kinetic barriers to SNAREpin assembly in the regulation of membrane docking/priming and fusion. Proceedings of the National Academy of Sciences of the United States of America 113:10536-10541.

25. Pierce, M. M., C. S. Raman, and B. T. Nall. 1999. Isothermal titration calorimetry of protein-protein interactions. Methods 19:213-221.

26. Morrison, E. A., G. T. DeKoster, S. Dutta, R. Vafabakhsh, M. W. Clarkson, A. Bahl, D. Kern, T. Ha, and K. A. Henzler-Wildman. 2011. Antiparallel EmrE exports drugs by exchanging between asymmetric structures. Nature 481:45-50.

27. Weber, G. 1953. Rotational Brownian motion and polarization of the fluorescence of solutions. Advances in protein chemistry 8:415-459.

28. Albrecht, A. C. 1961. Polarizations and Assignments of Transitions - Method of Photoselection. J Mol Spectrosc 6:84-108.

29. Lakowicz, J. R. 2006. Principles of Fluorescence Spectroscopy. Springer.

30. Jakhanwal, S., C. T. Lee, H. Urlaub, and R. Jahn. 2017. An activated Q-SNARE/SM protein complex as a possible intermediate in SNARE assembly. The EMBO journal 36:1788-1802.

31. Kummel, D., S. S. Krishnakumar, D. T. Radoff, F. Li, C. G. Giraudo, F. Pincet, J. E. Rothman, and K. M. Reinisch. 2011. Complexin cross-links prefusion SNAREs into a zigzag array. Nature structural \& molecular biology 18:927-933.

32. Cho, R. W., L. K. Buhl, D. Volfson, A. Tran, F. Li, Y. Akbergenova, and J. T. Littleton. 2015. Phosphorylation of Complexin by PKA Regulates Activity-Dependent Spontaneous Neurotransmitter Release and Structural Synaptic Plasticity. Neuron 88:749-761.

33. Heyduk, T., and J. C. Lee. 1990. Application of fluorescence energy transfer and polarization to monitor Escherichia coli cAMP receptor protein and lac promoter interaction. Proceedings of the National Academy of Sciences of the United States of America 87:1744-1748.

34. Smith, P. K., R. I. Krohn, G. T. Hermanson, A. K. Mallia, F. H. Gartner, M. D. Provenzano, E. K. Fujimoto, N. M. Goeke, B. J. Olson, and D. C. Klenk. 1985. Measurement of protein using bicinchoninic acid. Analytical biochemistry 150:76-85.

35. Krohn, R. I. 2011. The colorimetric detection and quantitation of total protein. Current protocols in cell biology Appendix 3:3H.

36. Bradford, M. M. 1976. A rapid and sensitive method for the quantitation of microgram quantities of protein utilizing the principle of protein-dye binding. Analytical biochemistry 72:248-254. 


\section{Figure Legends}

Figure 1. SDS-PAGE analysis of purified proteins. (A) Illustration of the structure of the tSNARE. (B) SDS-PAGE gel image of the purified t-SNARE.

Figure 2. Water-water titration to test the ITC's noise and stability of baseline. (A) ITC baseline and the raw data in power for each injection during the water-water titration. (B) Power data after baseline subtraction vs. time for each injection from the same water-water titration was presented in the upper panels. Integrated heat of each injection normalized by the moles of injectant vs. the molar ratio was plotted in the lower panels.

Figure 3. ITC analysis of the thermodynamics of SNARE assembly. (A) through (C) ITC measurements of $\mathrm{Vc}, \mathrm{CDV}$, and $\mathrm{Vn}$ assembling with the t-SNARE, respectively. $\sim 220 \mu \mathrm{MVc}$ peptide was titrated into $\sim 22 \mu \mathrm{M}$ t-SNARE solution (A), $\sim 400 \mu M$ CDV peptide was titrated into $\sim 25 \mu \mathrm{M}$ t-SNARE solution (B), and $\sim 250 \mu M$ Vn peptide was titrated into $\sim 22 \mu \mathrm{M}$ t-SNARE solution (C). Raw data in power vs. time during the injection was presented in the upper panels. Integrated heat of each injection normalized by the moles of injectant vs. the molar ratio was plotted in the lower panels. The solid lines represented the best fit to the black squares obtained from a nonlinear least-squares fit assuming a simple one-site chemical reaction. Thermodynamic parameters for each binding reaction were listed in Table 1. (D) ITC measurements of Vn assembling with prebound t-SNARE. To prebind the Vc peptide, the t-SNARE and Vc peptide (molar ratio 1:8) were incubated together at $37^{\circ} \mathrm{C}$ for $60 \mathrm{~min}$ prior to titration. In ITC experiments, $\sim 200 \mu \mathrm{M}$ Vn peptide was titrated into $\sim 20 \mu \mathrm{M}$ prebound t-SNARE mixture. The solid lines represented the best fit to the black squares obtained from a nonlinear least-squares fit assuming a simple one-site chemical reaction. Thermodynamic parameters for each binding reaction were listed in Table 1. (E) ITC measurements of CDV assembling with prebound tSNARE. $\sim 450 \mu \mathrm{M}$ CDV peptide was titrated into $\sim 42 \mu \mathrm{M}$ prebound t-SNARE mixture. Thermodynamic parameters for each binding reaction were listed in Table 1. 
Figure 4. Fluorescence anisotropy assays of the thermodynamics and kinetics of SNARE assembly in the absence of pre-structuring. (A) through (C) Fluorescence anisotropy experiments were performed to monitor the process of $360 \mathrm{nM} \mathrm{Vn}$ peptide (A), $210 \mathrm{nM} \mathrm{Vc}$ peptide (B), or $210 \mathrm{nM} \mathrm{CDV} \mathrm{(C)} \mathrm{binding} \mathrm{to} \mathrm{the} \mathrm{cytosolic} \mathrm{t-SNARE} \mathrm{at} \mathrm{various} \mathrm{concentrations,}$ respectively, at $26^{\circ} \mathrm{C}$. The Vn peptide contains VAMP2 residues 1-57 S28C, Vc contains VAMP2 residues 58-94 and Cysteine residue, and CDV contains VAMP2 residues 1-94 S28C. All three peptides were labeled with Texas Red. (D) The normalized initial slopes of the binding process of Vn (pink squares), Vc (black diamonds) and CDV (green circles) to the t-SNARE at various concentrations were plotted versus the concentration of t-SNARE. The solid lines were fits according to the Equation 5 to obtain the on-rates. (E) Plateau anisotropy values of the binding process of Vn (pink squares), Vc (black diamonds) and CDV (green circles) to the t-SNARE at various concentrations were plotted versus the concentration of t-SNARE. The solid lines were fits using the Equation 8 to obtain the affinity constants.

Figure 5. Fluorescence anisotropy assays of the thermodynamics and kinetics of SNARE assembly in the presence of pre-structuring. (A) \& (B) Fluorescence anisotropy experiments were performed to monitor the process of $60 \mathrm{nM}$ Vn peptide (A), or $66 \mathrm{nM} \mathrm{CDV} \mathrm{(B)} \mathrm{binding} \mathrm{to}$ the cytosolic t-SNARE prebound with a Vc peptide at various concentrations, respectively, at $26^{\circ} \mathrm{C}$. The prebound t-SNARE was formed by incubating t-SNARE and Vc peptide (molar ratio Vc: t-SNARE $=8: 1$ ) for 60 minutes. (C) The normalized initial slopes of the binding process of $\mathrm{Vn}$ (pink squares), or CDV (green circles) to the t-SNARE prebound with a Vc peptide at various concentrations were plotted versus the concentration of t-SNARE. The solid lines were fits according to the Equation 5 to obtain the on-rates. (D) Plateau anisotropy values of the binding process of Vn (pink squares), or CDV (green circles) to the t-SNARE prebound with a Vc peptide at various concentrations were plotted versus the concentration of t-SNARE. The solid lines were fits using the Equation 8 to obtain the affinity constants.

Figure 6. Kinetic energy barriers of $\mathrm{N}$ - and $\mathrm{C}$ - terminal assemblies. (A) \& (B) ) Fluorescence anisotropy experiments were performed to monitor the binding process of Vn to the t-SNARE at various concentrations and different temperatures in the absence (green triangles) or presence of 
(red diamonds) prebinding with excessive $\mathrm{Vc}$, respectively. The on-rate, $k_{o n}$, and off-rate, $k_{\text {off }}$, were determined at each temperature, $T$. $k_{\text {on }}$ and $k_{\text {off }}$ were then plot versus $1 / T$ according to Arrhenius' equation to obtain the kinetic energy barriers of N-terminal association (A) and disassociation (B), respectively. (C) \& (D) Fluorescence anisotropy experiments were performed to monitor the binding process of $\mathrm{Vc}$ to the t-SNARE at various concentrations and different temperatures in the absence (blue circles) or presence of (black squares) prebinding with excessive $\mathrm{Vn}$, respectively. The on-rate, $k_{o n}$, and off-rate, $k_{\text {off, }}$, were determined at each temperature, $T . k_{o n}$ and $k_{\text {off }}$ were then plot versus $1 / T$ according to Arrhenius' equation to obtain the kinetic energy barriers of C-terminal association (C) and disassociation (D), respectively.

\section{Figure 7. Energy landscape of the kinetic barriers of the $\mathrm{N}$ - and $\mathrm{C}$ - terminal assemblies.}

Using $E_{a, o n}$ and $E_{a, o f f}$ of the N-terminal and C- terminal assemblies obtained above, an energy landscape of the $\mathrm{N}$ - to $\mathrm{C}$ - directional zippering pathway is determined. In the absence of activation of the t-SNARE through prebinding with Vc peptide, the zippering pathway follows the blue solid curve with a high barrier on the N-terminal assembly. In the presence of activation of the t-SNARE through prebinding with Vc peptide, the zippering pathway follows the black solid curve and the barrier of N-terminal assembly is substantially reduced. In both situations, the barrier of C-terminal assembly is low $\left(\sim 4.7 k_{B} T\right)$ as N-terminal zippering occurs first, which prestructures the C-terminus of the t-SNARE. An intermediate energy minimum exists between the $\mathrm{N}$ - and $\mathrm{C}$ - terminal assemblies. 


\section{Figures}

Figure 1

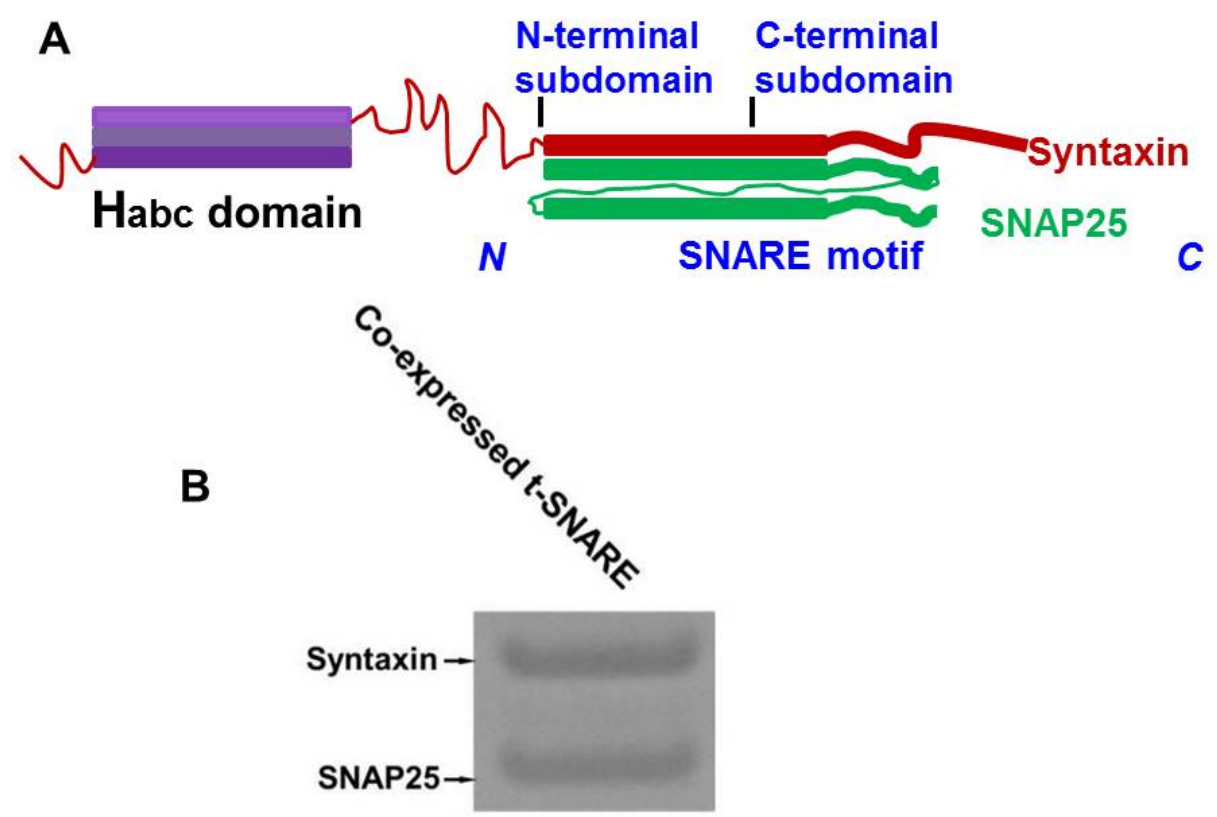


Figure 2
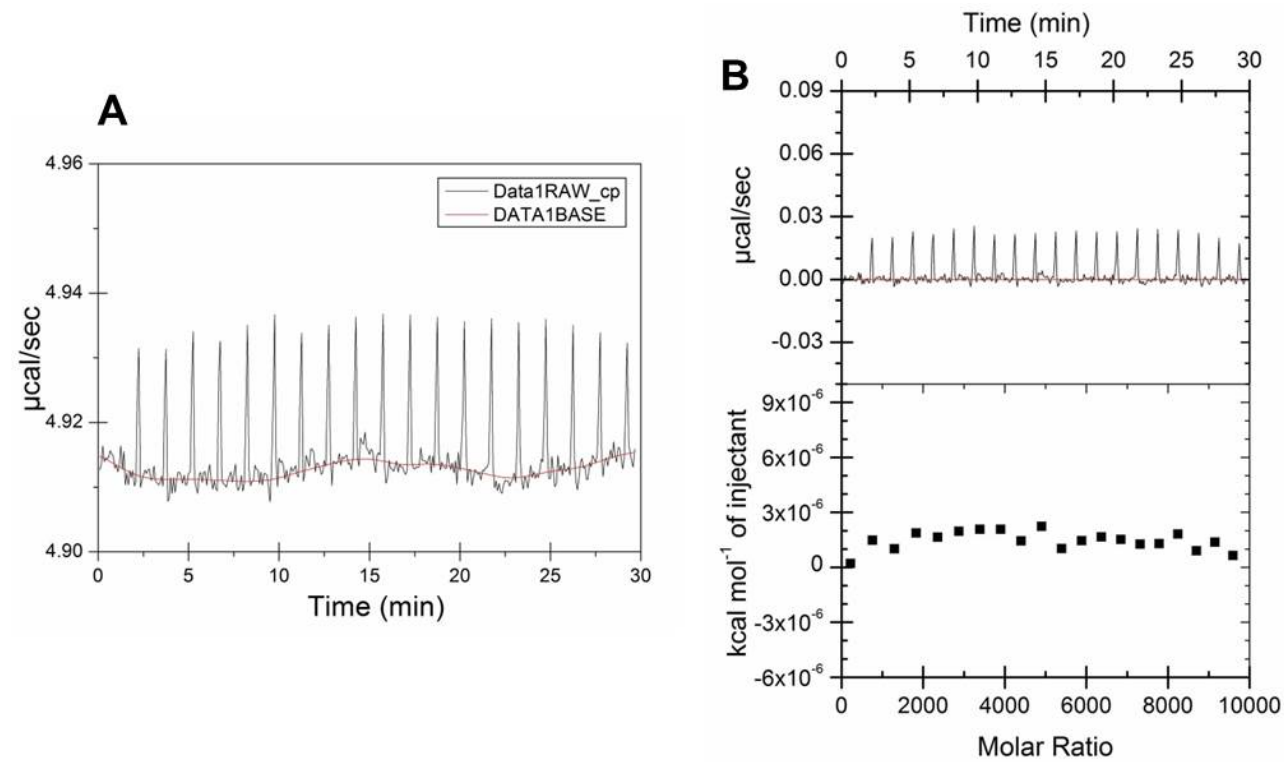
Figure 3
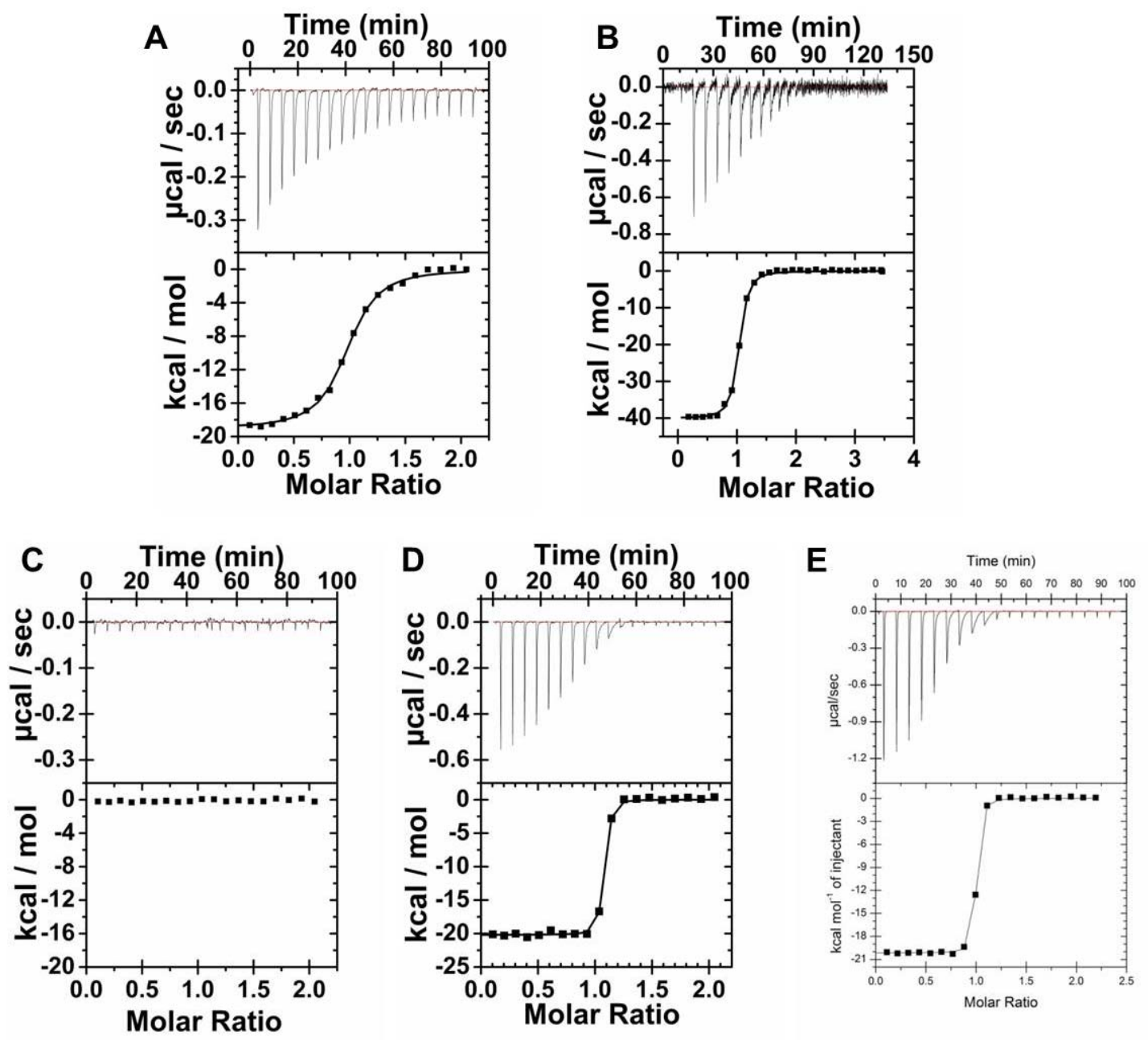
Figure 4
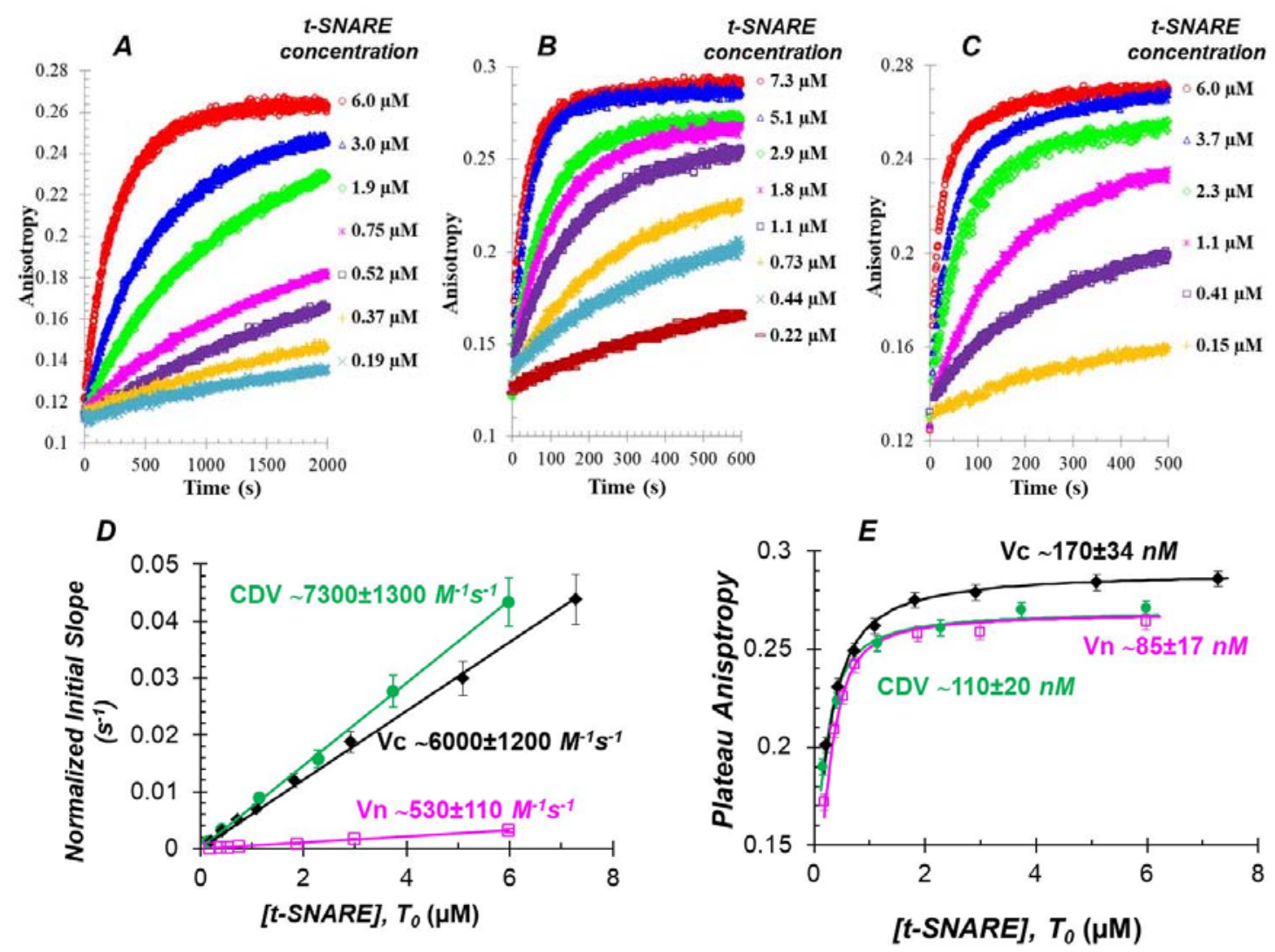
Figure 5
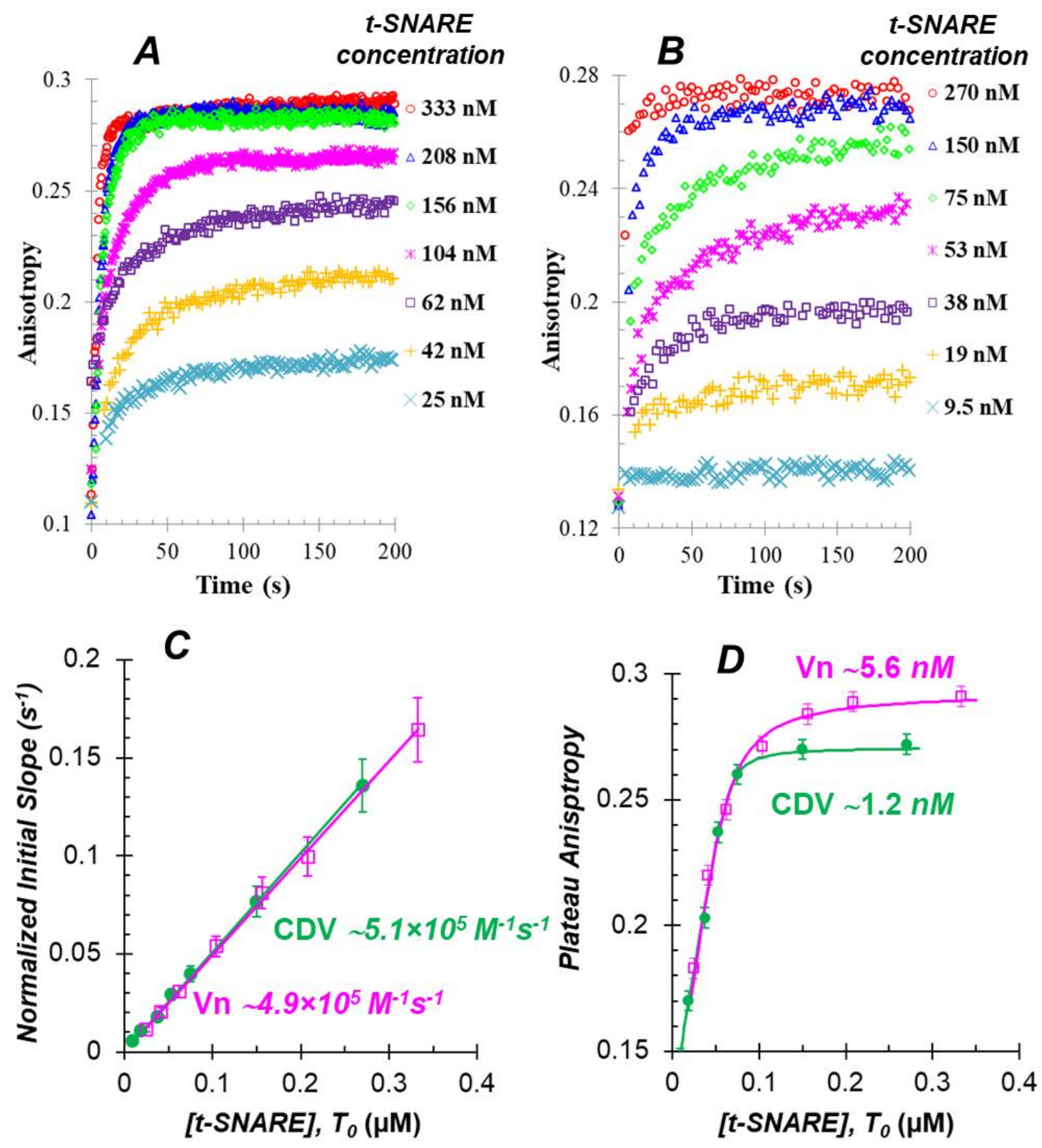
Figure 6
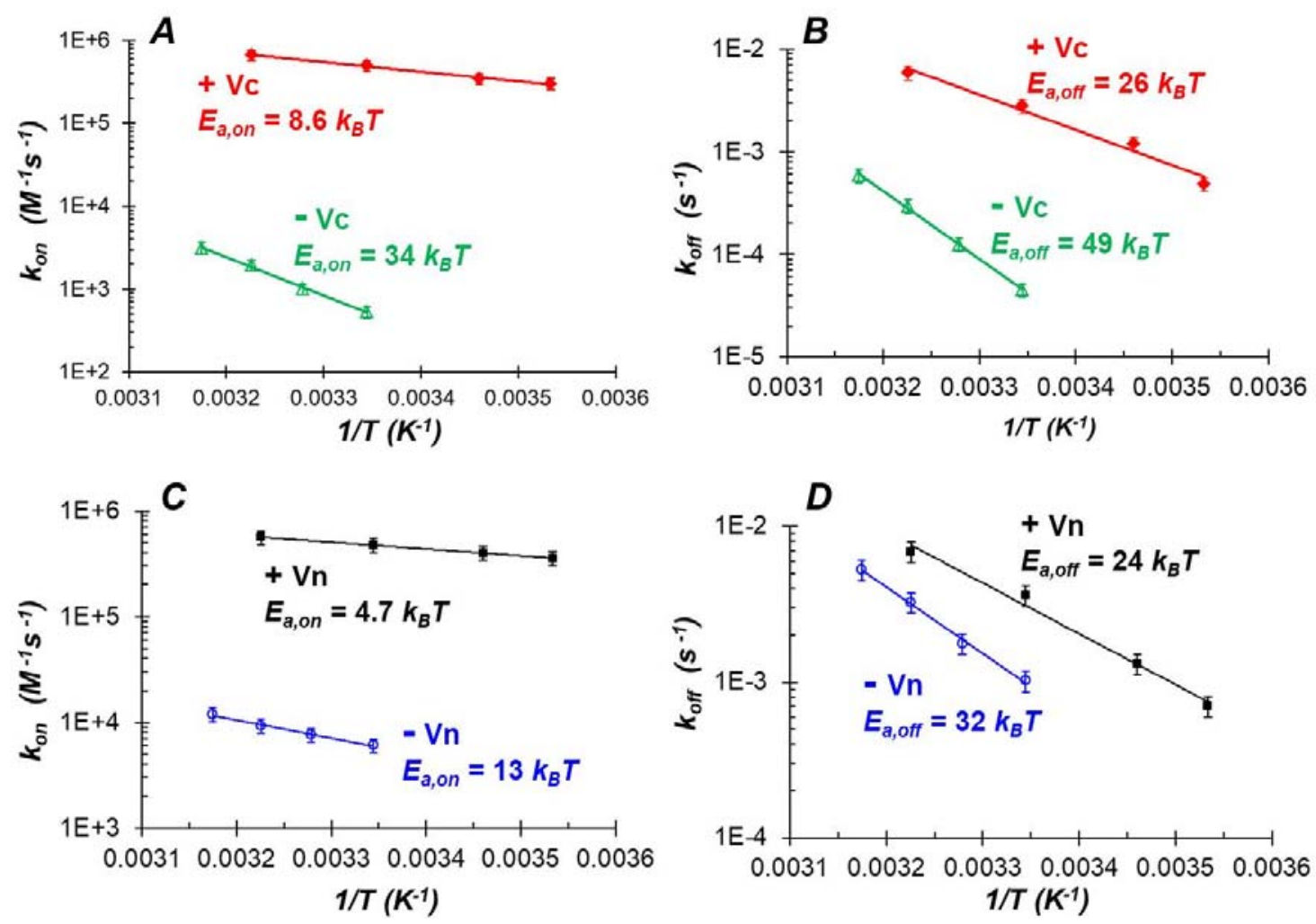
Figure 7

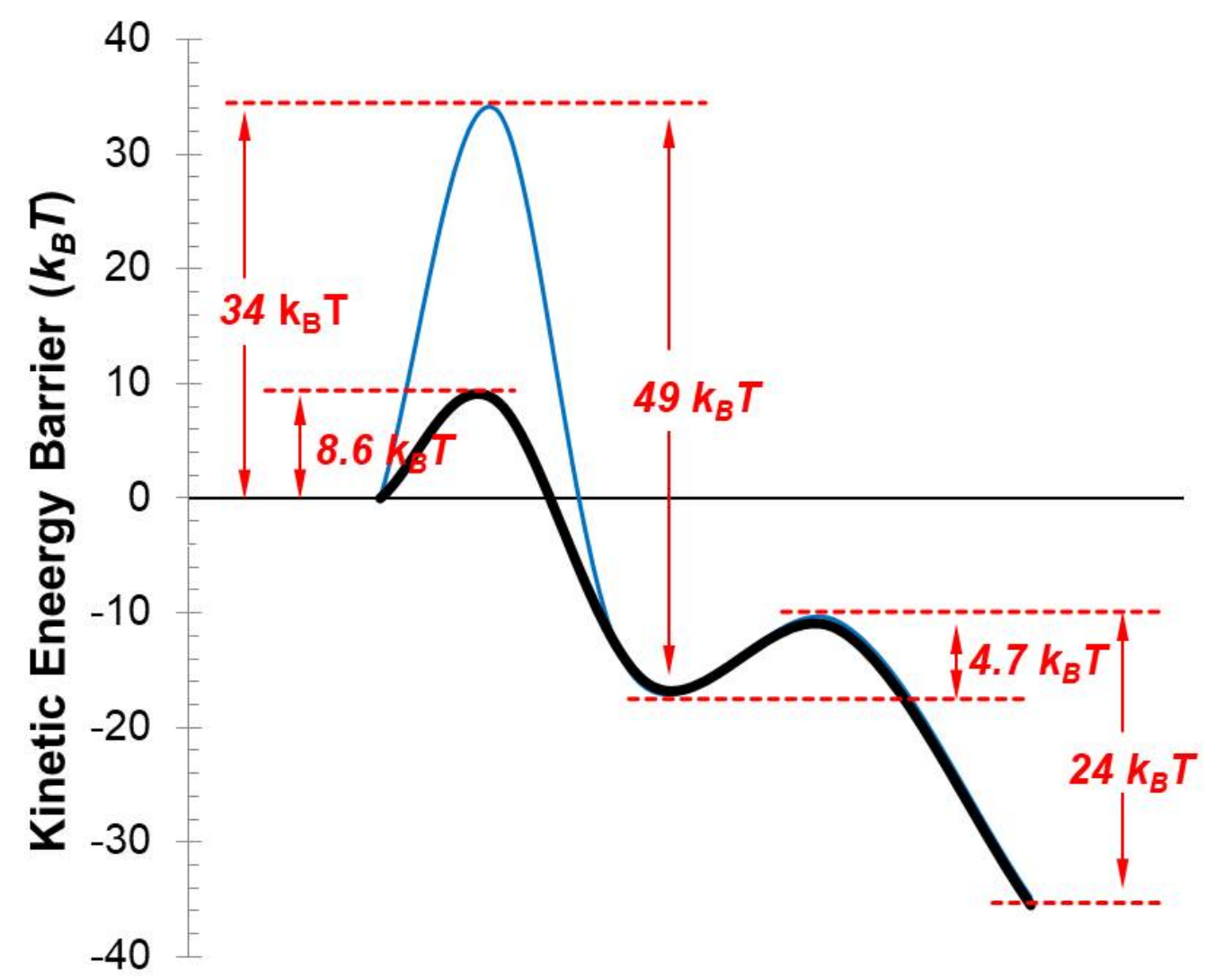


Table 1. Thermodynamic parameters of protein interactions from ITC measurements.

\begin{tabular}{|c|c|c|c|c|c|c|c|}
\hline Sample Cell & Titrant & $N$ & $\begin{array}{l}K_{D} \\
(n M)\end{array}$ & $\begin{array}{c}\Delta H \\
\left(\mathrm{kcal} \cdot \mathrm{mol}^{-1}\right)\end{array}$ & 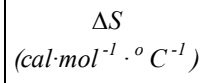 & $\begin{array}{c}\Delta G \\
\left(\mathrm{kcal} \cdot \mathrm{mol}^{-1}\right)\end{array}$ & $\begin{array}{c}\Delta G \\
\left(k_{B} T\right)\end{array}$ \\
\hline t-SNARE & Vn peptide & $\sim$ & $\sim$ & $\sim$ & $\sim$ & $\sim$ & $\sim$ \\
\hline t-SNARE & Vc peptide & $0.95 \pm 0.01$ & $444 \pm 47$ & $-19.1 \pm 0.2$ & $-34.9 \pm 0.9$ & $-8.7 \pm 0.1$ & $14.6 \pm 0.1$ \\
\hline $\mathrm{t}$-SNARE & $\mathrm{CDV}$ & $0.99 \pm 0.01$ & $132 \pm 10$ & $-40.1 \pm 0.2$ & $-97.8 \pm 0.8$ & $-9.8 \pm 0.1$ & $15.8 \pm 0.1$ \\
\hline $\begin{array}{l}\text { t-SNARE pre-incubated } \\
\text { with Vc peptide }\end{array}$ & Vn peptide & $1.03 \pm 0.01$ & $6.8 \pm 1.3$ & $-20.2 \pm 0.1$ & $-30.3 \pm 0.7$ & $-11.2 \pm 0.2$ & $18.8 \pm 0.2$ \\
\hline $\begin{array}{l}\text { t-SNARE pre-incubated } \\
\text { with Vc peptide }\end{array}$ & $\mathrm{CDV}$ & $0.96 \pm 0.01$ & $15 \pm 3$ & $-20.2 \pm 0.1$ & $-31.8 \pm 0.8$ & $-10.7 \pm 0.1$ & $18.0 \pm 0.2$ \\
\hline $\begin{array}{l}\text { t-SNARE pre-incubated } \\
\text { with Vn peptide }\end{array}$ & Vc peptide & $1.04 \pm 0.01$ & $56 \pm 9$ & $-18.9 \pm 0.2$ & $-30.0 \pm 1.2$ & $-9.9 \pm 0.1$ & $16.7 \pm 0.2$ \\
\hline
\end{tabular}

